\title{
Feature Tracking Algorithm for Circumferential Strain using High Frame Rate Echocardiography
}

\author{
Martin V Andersen ${ }^{1}$, Cooper Moore ${ }^{2}$, Samuel E Schmidt ${ }^{1}$, Peter Søgaard ${ }^{3}$, Johannes J Struijk ${ }^{1}$, \\ Joseph Kisslo ${ }^{4}$,Olaf T von Ramm ${ }^{2}$ \\ ${ }^{1}$ Aalborg University, Aalborg, Denmark. ${ }^{2}$ Duke University, Durham, USA. \\ ${ }^{3}$ Aalborg University Hospital, Aalborg, Denmark. ${ }^{4}$ Duke University Hospital, Durham, USA.
}

\begin{abstract}
This study aims to describe a feature tracking algorithm tailored to estimate circumferential strain on high frame rate ultrasound (HFR-US) images. The algorithm used the Hungarian assignment algorithm for tracking a basic feature descriptor. A second order Kalman model was used to recursively describe regional myocardial displacement along the myocardial contour, and the strain was calculated using these displacements. HFR-US images at 360 fps were acquired from a patient with left bundle branch block (LBBB) in the parasternal short axis view with a biventricular (BiV) pacer turned off and on. There was a large variation in the onset and end of mechanical contraction for each individual myocardial region when the BiV pacer was turned off. The variation reduced immediately when the BiV pacer was turned on. The presented algorithm can estimate circumferential strain using high frame rate ultrasound images. The circumferential strain does suffer from high intra- and inter-operator variance due to the lack of landmarks making it difficult to reproduce results. However, as circumferential and longitudinal strain offer two different, but somehow interrelated, descriptors of complex mechanical movement of the heart. The observed variance reduction may be indicative for a positive $B i V$ response in $L B B B$ patients.
\end{abstract}

\section{Introduction}

Cardiac deformation imaging using high frame rate (HFR) ultrasound (US) images can give clinicians a new diagnostic tool for evaluating cardiac health.

The frame rate (FR) of conventional ultrasound is not adequate for appreciating the electromechanical coupling and other propagating mechanical and electrical events in the myocardium. In order to appreciate these events a temporal resolution of close to $2 \mathrm{~ms}$ would be necessary [1]. In the last decade propagating events have been noted in vivo using different US modalities such as Tis- sue Doppler Imaging (TDI) and B-mode [2-10]. These studies all use velocity curves to describe the electromechanical coupling. However, velocity measurements do not discriminate between overall cardiac motion and local myocardial contraction. To appreciate local myocardial contraction clinical cardiac strain measurements is commonly used [11]. Conventional frame rate longitudinal strain is the most commonly used and has been implemented as an independent predictor for cardiac resynchronization therapy (CRT) responders [11]. Previously an algorithm for describing longitudinal strain using HFR-US images has been described [12]. For a more complete description of contraction it may be valuable to measure circumferential cardiac strain in HFR-US images as well. Some studies suggest that circumferential strain has better predictive value, while other studies suggested that circumferential strain has weaker predictive value than radial strain $[13,14]$. We present a new method to help with the assessment of the the value of circumferential strain measurements. Here the HFR-US longitudinal strain algorithm described in [12] is extended to estimate strain curves to circumferential strain using the parasternal Short Axis (PSAX) view.

\section{Methods}

\subsection{Data}

HFR-US images were acquired using an experimental phased array US scanner, T5 developed at Duke University (T5). The T5 can acquire US images of the heart at $360 \mathrm{fps}$ with a scan depth of $12 \mathrm{~cm}$ to $14 \mathrm{~cm}$ and a field of view (FOV) of $80^{\circ}$ using a $3.5 \mathrm{MHz}$ transducer while showing live images on a monitor while acquiring. Subject data is acquired under the Institutional review board (IRB) approval with written informed consent from the patient by an independent recruiter. The subject was imaged on the T5 system at a acquisition rate of $360 \mathrm{fps}$ in the PSAX view. A certified clinician turned the implanted biventric- 
ular (BiV) pacer off while another PSAX image was acquired.

\subsection{Algorithm}

The algorithm is designed to analyze HFR-US images and estimate strain curves by tracking motion. This is done by tracking small translations between frames using features and estimate strain curves along the myocardial wall contour. It is possible to estimate both longitudinal and circumferential strain using the algorithm, and it is described in 5 stages which are explained below.

Stage 1 Filtering: As US images contain a significant amount of information that does not relate to motion and make motion estimation difficult.. High frequency temporal noise was removed using a simple first order recursive filter to get a filtered image $(\hat{I}(x, y, t))$ by using Equation 1.

$$
\hat{I}(x, y, t)=\alpha_{t} \times \hat{I}(x, y, t-1)+\left(1-\alpha_{t}\right) \times I(x, y, t)
$$

Where $\alpha_{t}$ is an integration constant taking a value between 0 and $1, I(x, y, t)$ is the US image in frame $t$ and $\hat{I}(x, y, t-1)$ is the filtered image of the previous frame $t-1$. For this version $\alpha_{t}=0.5$. To improve the extraction of features a spatial filter is also used. A Gaussian spatial filter is implemented using a $3 \times 3$ pixel window. Because US sampling varies with respect to depth in Cartesian coordinates and is not as constant as in spherical coordinates the filter is implemented in spherical coordinates.

Stage 2 Feature Detection and Extraction: When estimating motion one option is to only evaluate pixels in the images where there is a higher likelihood to estimate motion correctly. Feature Extraction is based on finding particular pixels $(\mathrm{p}(\rho, \theta, \mathrm{t}))$, where $\rho$ is the scan depth, $\theta$ is the azimuth angle and $t$ is the frame, which are best suited for motion detection.

Feature Extraction is based on developing a suitable numeric description (Descriptor) in terms of a neighborhood of $\mathrm{p}(\rho, \theta, \mathrm{t})$ to qualify the feature. There are multiple sophisticated descriptors available such as SURF, HOG or FREAK [15-17]. But, if the translations are small sophisticated descriptors are not necessary as long as the frame to frame translation has a maximum limit. In this algorithm the feature is defined by the largest pixel intensity within a $3 \times 3$ pixel neighborhood. The intensity of the particular pixel $\mathrm{p}(\rho, \theta, \mathrm{t})$ is extracted and used as a descriptor.

Stage 3 Feature Tracking: After extracting features, the features are tracked by linking individual features between frames using the feature descriptor. As this can be considered an assignment problem the Hungarian algorithm is implemented to assign features to feature tracks [18]. The absolute intensity ratio between features in frame $t$ and $t-1$ in decibel $(\mathrm{dB})$ is used as the cost matrix. Furthermore if the intensity difference between features is above $6 \mathrm{~dB}$ or translation is above the maximum frame to frame translation $\left(T \times v_{\max } \times 1.25\right.$ where $T$ is temporal resolution ( $T=1 / \mathrm{FR}$ ) and $v_{\max }$ is $240 \mathrm{~mm} / \mathrm{s}$ which is the maximum velocity of a healthy myocardial wall as estimated by TDI) the cost of linking the two features will be set to infinity [19].

Stage 4 Region Tracking: Out of plane motion and decorrelation of speckle that happens as a function of distance makes it improbable to track individual features through an entire cardiac cycle [20]. Instead the bulk displacement of feature tracks within a larger area are combined into a continuous track that describe the myocardial displacement $\left(\mathrm{X}_{\mathrm{n}}\right)$. The initial points $\left\{\mathrm{X}_{1}(1), \ldots, \mathrm{X}_{\mathrm{B}}(1)\right\}$ and width of the myocardium is defined by manually marking the mid myocardium along the entire contour and the thickness region in the myocardium $\left(\mathrm{L}_{\mathrm{T}}\right)$. An ellipsoid is fitted to the points and divided into arbitrary number of points (B) which are evenly divided with respect to angle where the right ventricular free wall is used as an anatomical reference. In order to estimate the bulk of displacement a weighted average is calculated for all feature tracks within a $\mathrm{L}_{\mathrm{T}} / 2$ radius of $\mathrm{X}_{\mathrm{n}}(\mathrm{t})$. First, a Gaussian weight $\left(w_{G}\right)$ was calculated for each feature track, depending on the distance to $\mathrm{X}_{\mathrm{n}}$ as defined by Equation 2 .

$$
\mathrm{w}_{\mathrm{G}}(\mathrm{j})=\frac{1}{2 \pi \sigma} \mathrm{e}^{\frac{-\mathrm{d}\left(\mathrm{x}_{\mathrm{j}}(\mathrm{t}), \mathrm{x}_{\mathrm{n}}(\mathrm{t}-1)\right)^{2}}{2 \sigma^{2}}}
$$

Where $d\left(x_{j}(t), X_{n}(t-1)\right)$ is the Euclidean distance between the feature $\left(\mathrm{x}_{\mathrm{j}}(\mathrm{t})\right)$ and $\mathrm{X}_{\mathrm{n}}(\mathrm{t}-1)$ and $\sigma=\sqrt{\mathrm{L}_{\mathrm{T}}}$. Second, the inverse directionality difference weight $\left(\mathrm{w}_{\mathrm{D}}\right)$ of the feature tracks with respect to the bulk displacement of a $22 \mathrm{~ms}$ window $\left(\tau_{\mathrm{w}}\right)$ as defined by Equation 3 .

$$
\mathrm{w}_{\mathrm{D}}(\mathrm{j})=\frac{1}{\mathrm{~d}\left(\frac{\mathrm{d}}{\mathrm{d} \tau_{\mathrm{w}}} \mathrm{x}_{\mathrm{j}}(\mathrm{t}), \widetilde{\mathrm{d} \tau_{\mathrm{w}}} \mathrm{X}_{\mathrm{n}}(\mathrm{t}-1)\right)}
$$

Where $\frac{d}{d \tau_{\mathrm{w}}} \mathrm{x}_{\mathrm{j}}$ is the directional components of feature track $j$ and $\frac{\mathrm{d}}{\mathrm{d} \tau_{\mathrm{w}}} \mathrm{X}_{\mathrm{n}}$ is the median of the directional components of all feature tracks in a specific region. $\mathrm{w}_{\mathrm{G}}$ and $\mathrm{w}_{\mathrm{D}}$ are both normalized so $\sum \mathrm{w}_{\mathrm{G}}=\sum \mathrm{w}_{\mathrm{D}}=1$. The estimated component (Y) is calculated using Equation 4.

$$
\begin{aligned}
& \mathrm{Y}=\mathrm{X}_{\mathrm{n}}(\mathrm{t}-1)+ \\
& \sum_{j=1}^{J}\left(\frac{\mathrm{d}}{\mathrm{dt}} \mathrm{x}_{\mathrm{j}}(\mathrm{t}) \times\left(\frac{\alpha_{\mathrm{D}}}{\alpha_{\mathrm{D}}+\alpha_{\mathrm{G}}} \mathrm{w}_{\mathrm{D}}(\mathrm{j})+\frac{\alpha_{\mathrm{G}}}{\alpha_{\mathrm{D}}+\alpha_{\mathrm{G}}} \mathrm{w}_{\mathrm{G}}(\mathrm{j})\right)\right)
\end{aligned}
$$

Where $\alpha_{D}$ and $\alpha_{G}$ are the confidence in each of the weights. For this version $\alpha_{D}=\alpha_{G}=1 . \mathrm{X}_{\mathrm{n}}(\mathrm{t})$ is then updated recursively using using a second order Kalman model and $\mathrm{Y}$ as the new measured state. At the end of each 
(a)

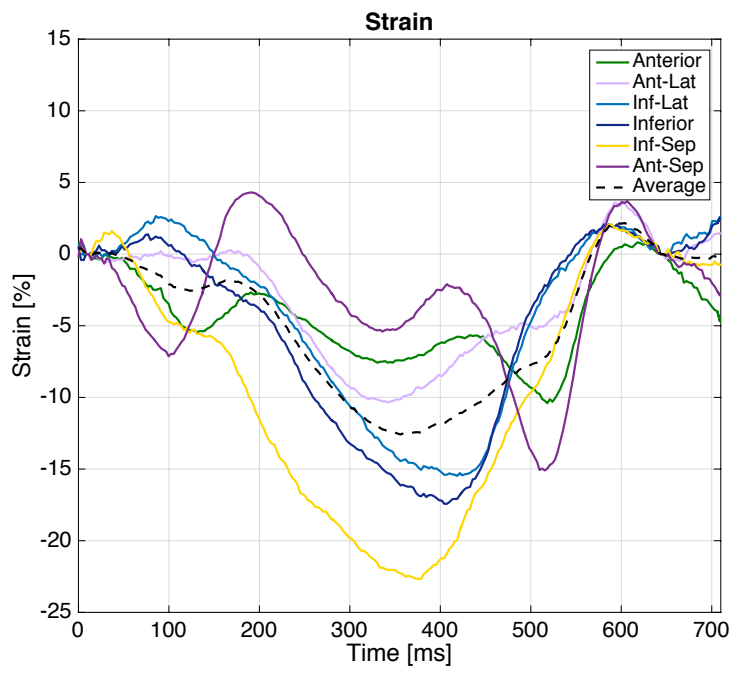

(b)

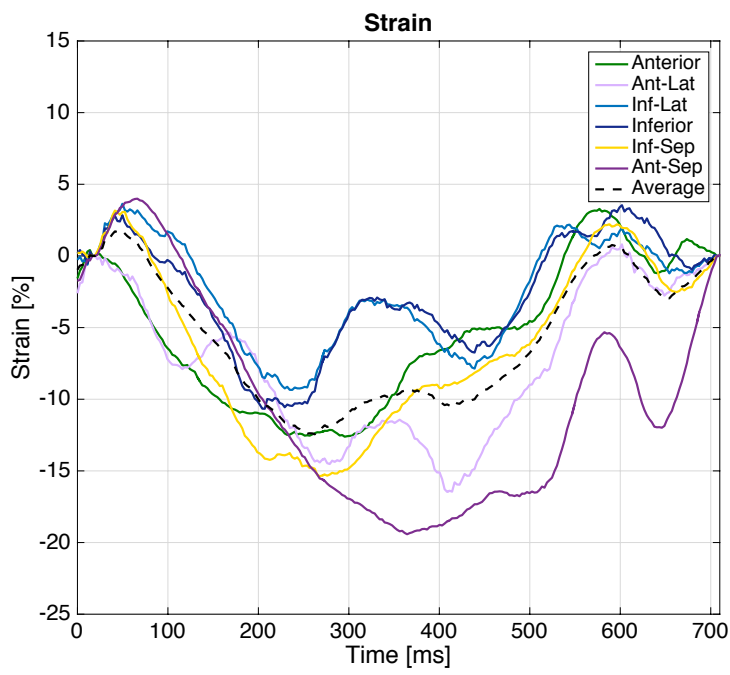

Figure 1: Circumferential strain is described as a function of time with the biventricular $(\mathrm{BiV})$ pacer turned off in Subfigure (a) and turned on in Subfigures (b). The legend describe the myocardial subregion of where the strain curves are estimated from, etc. Ant-Lat refer to the anterior lateral wall, and Inf-Sep refer to the inferior septal wall.

cardiac cycle drift compensation is calculated by multiplying a constant variable at all times $t^{\prime}$ where $X_{n}\left(t^{\prime}\right)$ have the opposite direction of the drift component.

Stage 5 Strain Estimation: Cardiac strain $(\varepsilon(t))$ is calculated to describe the contraction between two points independent of overall cardiac motion. $\varepsilon(t)$ is the percentage change in distance with respect to an initial condition, which is defined by Equation 5 [21].

$$
\varepsilon(t)=\frac{L(t)-L(1)}{L(1)} \times 100 \%
$$

Where $L(t)$ is the Euclidean distance between multiple region tracks $\left\{\mathrm{X}_{1}, \ldots, \mathrm{X}_{\mathrm{B}}\right\}$.

\section{Results}

Figures 1(a-b) show circumferential strain curves derived from US images using the PSAX view from a 71 year old male with LBBB and a biventricular (BiV) pacer. US images were acquired on the same day and show how mechanical contraction differs between the $\mathrm{BiV}$ pacer being turned off and on respectfully.

A measure from maximum myocardial stretching to end of the plateau immediately before mechanical contraction onset $\left(t_{\text {onset }}\right)$ and the first minimum nadir after mechanical contraction onset $\left(t_{\text {contract }}\right)$ was manually measured, see Table 1.

\begin{tabular}{|c||c||c|c|}
\hline & $t_{\text {onset }}[\mathrm{ms}]$ & $t_{\text {contract }}[\mathrm{ms}]$ & $t_{\text {onset }}-t_{\text {contract }}[\mathrm{ms}]$ \\
\hline \hline $\mathrm{BiV}_{\text {off }}$ & $89 \pm 69$ & $312 \pm 144$ & 222 \\
\hline $\mathrm{BiV}_{\text {on }}$ & $-9 \pm 20$ & $211 \pm 55$ & 220 \\
\hline
\end{tabular}

Table 1: Timing results.

Figure 1(a) show that the myocardial segments do not synchronously contact when the $\mathrm{BiV}$ pacer off, and that with the BiV pacer on the mechanical contraction occurs more synchronously, see Figure 1(b). The average time between $t_{\text {onset }}$ and $t_{\text {contract }}$ only differed by $4 \mathrm{~ms}$, see Table 1. Furthermore, both $t_{\text {onset }}$ and $t_{\text {contract }}$ with the BiV pacer off had a higher variation than with the BiV pacer on, see Table 1.

\section{Discussion}

In this paper a feature tracking algorithm for estimating circumferential strain on high frame rate ultrasound images is demonstrated. While sophisticated feature descriptors such as SURF, HOG or FREAK can be used, a high frame rate makes it possible to describe a feature adequately to detect them in adjacent frames using only basic descriptors. The weighted average algorithm and the Kalman model makes new feature descriptors easy to implement without changing other stages of the algorithm. When a $\mathrm{BiV}$ pacer is turned on, the cardiac contraction is more synchronous as compared to when it is turned off, see Table 1. The average rapid contraction between $t_{\text {onset }}$ and $t_{\text {contract }}$ is almost identical though they were delayed in time with the BiV pacer off, which could be due to the detour of the electrical excitation through the right bundle causing a delay. This support the assumption that it is an electrical obstruction, and not a mechanical problem. Previous studies are inconsistent with respect to which view gives the best predictive value with responders to CRT $[11,13,14]$. It is important to remember that 
the three dimensional cardiac contraction map is not adequately described by a single view. The PSAX view suffer from high intra- and inter-operator variance due to the lack of good structures as do the AP4 chamber making it difficult to reproduce results. However, the AP4 and PSAX offer two different, but somehow interrelated, descriptors of complex mechanical movement of the heart. The presented algorithm can estimate circumferential strain using high frame rate ultrasound images.

\section{Acknowledgments}

HFR-US images were acquired at the Duke University Medical Center in the Cardiac Diagnostic Unit, of the Division of Cardiology in association with the Pratt School of Engineering, Department of Biomedical Engineering, Duke University.

\section{References}

[1] Cikes M, D’Hooge J. Ultrafast cardiac ultrasound imaging: Technical principles, applications, and clinical benefits. JACC Cardiovascular Imaging 2014;7(8):812-823. ISSN 18767591.

[2] Kanai H. Propagation of Vibration Caused by Electrical Excitation in the Normal Human Heart. Ultrasound in Medicine and Biology 2009;35(6):936-948. ISSN 03015629.

[3] Provost J, Konofagou EE. Electromechanical Wave Imaging of Normal and Ischemic Hearts In Vivo. IEEE Transactions on Medical Imaging 2010;29(3):625-635. ISSN 0278-0062.

[4] Provost J, Konofagou EE. Electromechanical wave imaging for arrhythmias. Physics in medicine and biology 2011; 56(22):L1-11. ISSN 1361-6560.

[5] Lee WN, Tanter M. Mapping myocardial fiber orientation using echocardiography-based shear wave imaging. IEEE Transactions on Medical Imaging 2012;31(3):554562. ISSN 02780062.

[6] Song P, Chen S. Improved Shear Wave Motion Detection Using Pulse-Inversion Harmonic Imaging with a Phased Array Transducer. IEEE transactions on medical imaging 2013;32(12):2299-2310. ISSN 1558-254X.

[7] Brekke B, Aase SA. Ultra-high frame rate tissue doppler imaging. Ultrasound in Medicine and Biology 2014; 40(1):222-231. ISSN 0301-5629.

[8] Papadacci C, Tanter M. High-contrast ultrafast imaging of the heart. IEEE Transactions on Ultrasonics Ferroelectrics and Frequency Control 2014;61(2):288-301. ISSN 08853010.

[9] Pislaru C, Pislaru SV. Wave propagation of myocardial stretch: Correlation with myocardial stiffness. Basic Research in Cardiology 2014;109(6). ISSN 14351803.

[10] Tong L, D'hooge J. Wide-angle tissue doppler imaging at high frame rate using multi-line transmit beamforming: An experimental validation In Vivo. IEEE Transactions on Medical Imaging 2016;35(2):521-528. ISSN 1558-254X.
[11] Risum N, Kisslo J. Left bundle-branch block: The relationship between electrocardiogram electrical activation and echocardiography mechanical contraction. American Heart Journal 2013;166(2):340-348. ISSN 00028703.

[12] Andersen MV, Olaf. High frame rate deformation imaging in two dimensions using continuous speckle-feature tracking. Ultrasound in Medicine Biology Under Review;

[13] Helm RH, Leclercq C, Paris OP, Ozturk C, McVeigh E, Lardo AC, Kass DA. Cardiac dyssynchrony analysis using circumferential versus longitudinal strain: Implications for assessing cardiac resynchronization. Circulation 2005; 111(21):2760-2767. ISSN 00097322.

[14] Delgado V, Ypenburg C, van Bommel RJ, Tops LF, Mollema Sa, Marsan NA, Bleeker GB, Schalij MJ, Bax JJ. Assessment of Left Ventricular Dyssynchrony by Speckle Tracking Strain Imaging. Journal of the American College of Cardiology 2008;51(20):1944-1952. ISSN 07351097.

[15] Bay H, Ess A, Tuytelaars T, Van Gool L. Speeded-Up Robust Features (SURF). Computer Vision and Image Understanding jun 2008;110(3):346-359. ISSN 10773142.

[16] Dalal N, Triggs B. Histograms of Oriented Gradients for Human Detection. In 2005 IEEE Computer Society Conference on Computer Vision and Pattern Recognition (CVPR'05), volume 1. IEEE. ISBN 0-7695-2372-2. ISSN 1063-6919, 2005; 886-893.

[17] Alahi A, Ortiz R, Vandergheynst P. FREAK: Fast Retina Keypoint. In 2012 IEEE Conference on Computer Vision and Pattern Recognition. IEEE. ISBN 978-1-4673-1228-8, jun 2012; 510-517.

[18] Kuhn HW. The Hungarian method for the assignment problem. In 50 Years of Integer Programming 1958-2008: From the Early Years to the State-of-the-Art. Naval Research Logistics Quarterly. ISBN 9783540682745, 2010; 29-47.

[19] Oki T, Tabata T, Mishiro Y, Yamada H, Abe M, Onose Y, Wakatsuki T, Iuchi A, Ito S. Pulsed tissue doppler imaging of left ventricular systolic and diastolic wall motion velocities to evaluate differences between long and short axes in healthy subjects. Journal of the American Society of Echocardiography 1999;12(5):308-313.

[20] Trahey GE, Smith SW, Von Ramm T. Speckle pattern correlation with lateral aperture translation: experimental results and implications for spatial compounding. Ultrasonics Ferroelectrics and Frequency Control IEEE Transactions on 1986;33(3):257-264.

[21] Voigt JU, aolo Badano LP. Definitions for a common standard for 2D speckle tracking echocardiography: consensus document of the EACVI/ASE/Industry Task Force to standardize deformation imaging. European heart journal cardiovascular Imaging 2015;16(1):1-11. ISSN 20472412.

Address for correspondence:

Martin Vandborg Andersen

Fredrik Bajers Vej 7 C2-203

9000 Aalborg, Denmark

mvan@hst.aau.dk 